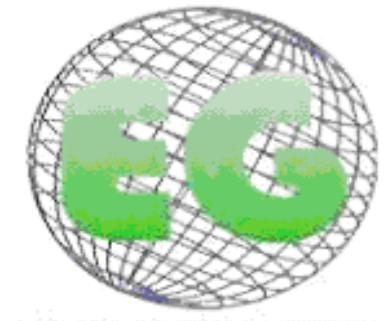

ISSN 1695-6141 N'25
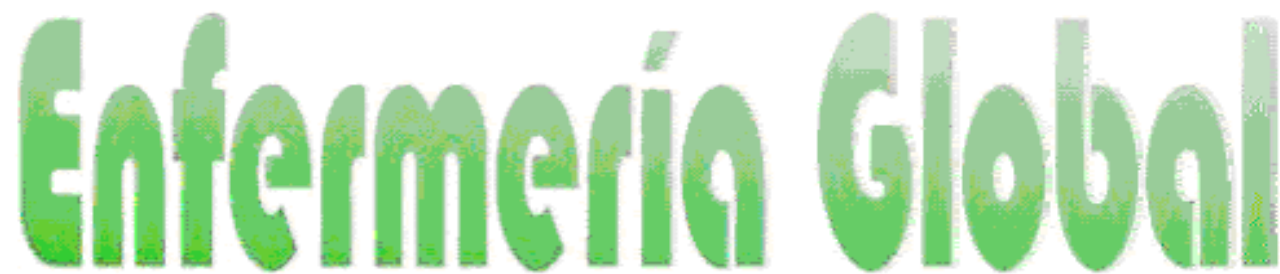

Revista electrónica trimestral de Enfermería

Enero 2012

www.um.es/egloball

\title{
Recuperación de la salud del cuidador principal, en proyecto sociocultural "Quisicuaba". Los Sitios, Centro Habana
}

Recovery of the health of the Main Carer in the "Quisicuaba" socio-cultural project. Los Sitios, Havana.

\section{*Martínez Cepero, Félix E.}

\author{
*MsC. Epidemiología. MsC. Salud Medio ambiental. Profesor Auxiliar de Enfermería Familiar y Social, \\ Psicología General y Social. Facultad de Ciencias Médicas Finlay-Albarrán. La Habana. Cuba.
}

Palabras clave: cuidador principa; enfermedad de Alzheimer; proyecto sociocultural

Keywords: main carer; Alazheimer' s disease; socio-cultural project

\section{RESUMEN}

La demencia causa discapacidad grave entre las personas que la padecen, provocando efectos diversos en las familias, y en la persona cuidadora, al extremo de experimentar estrés, frustración y total agotamiento. Comprobar la utilidad de un programa de recuperación de salud con un diseño descriptivo de corte transversal dentro del proyecto sociocultural "Quisicuaba, a través de una muestra de 26 cuidadores principales, permitió determinar características sociodemográficas y la persistencia de ansiedad, depresión y sobrecarga en estas personas, alteraciones posteriormente modificadas, luego de la intervención. Instrumentos diagnósticos como Inventario de ansiedad, escala de depresión de Hamilton, Cuestionario de Sobrecarga Zarit-Zarit, dinámicas grupales y entrevistas aportaron datos e interés, puesto que la tarea desarrollada permitió reconocer la creación de un espacio de respiro para estas personas, demostrando su utilidad con un mínimo de recursos y el apoyo de profesionales y aficionados del territorio. Solicitándose su generalización, a criterio de los participantes.

\begin{abstract}
Dementia leads to serious discapacities in its sufferers and can produce a variety of effects in the families and in the carer, who may experience stress, frustraton and total exhaustion. Assesing the usefulness of a crosssectional descriptive health recovery programme within the socio-cultural project, "Quiscuaba", using a sample of 26 main carers revealed certain socio-demographic characteristics - the persistence of anxiety, depression and burden in these people, which were modified after treatment. Diagnosis tools like the Hamilton depression scale, the Zarit-Zarit burden scale, group dynamics and interviews provided data of interest since the work performed supposed the creation of a breathing space, so demonstrating its worth, with a minimum use of professional and voluntary resources and support. Participants called for it to become more widespread.
\end{abstract}




\section{INTRODUCCIÓN}

Se estima que en el mundo unos 18 millones de personas padecen demencia, de ellas más de la mitad, son casos diagnosticados como demencia tipo Alzheimer señalándose la probabilidad de que una persona pueda desarrollar una demencia en la medida que aumenta la edad, por lo que es de prever que el número de casos de demencia aumentará rápidamente con el envejecimiento poblacional mundial en los próximos decenios.

La demencia causa discapacidad grave entre las personas que la padecen, provocando efectos diversos en las familias y en los amigos cercanos que se relacionan con la persona querida, al extremo de experimentar estrés, frustración y agotamiento, además de sentimientos de pérdida de la persona que antes conocían, sumado a elevados costos económicos que no siempre están disponibles para estos fines. ${ }^{(2,3)}$

El cuidado informal, definido como aquella prestación de cuidados a personas dependientes (ancianos, enfermos crónicos, discapacitados, etc.) por otra parte de la familia, (hijos/as, hermanos/as, esposo/a), los amigos, vecinos u otras personas que no reciben retribución económica por la ayuda que ofrecen es hoy una de las formas de trabajo más abnegadas y repetidas que suelen acometerse en nuestras comunidades, respondiendo a la necesidad e importancia de asistir a quienes ya no pueden valerse por sí mismos, reportando una mejora en la calidad de vida de la persona limitada respecto a la que ofrece la institucionalización, reduciendo de una manera sensible los costes para los servicios públicos sociales y sanitarios $^{(4,5)}$

Sin embargo, es importante reconocer que dentro de la familia, la prestación de cuidados no acostumbra a repartirse por igual entre sus miembros. Hay un "cuidador principal", sobre el que recae la mayor responsabilidad de los cuidados, en su mayoría sin una experiencia previa, un conocimiento o la guía de una persona competente para orientarle, entregando en ese intento, su salud y su vida. ${ }^{(5)}$

La asunción de esta nueva faena de manera permanente y sin el apoyo necesario conducirá de manera inmediata a la aparición de serios problemas en las relaciones familiares, fuertes reacciones emocionales, aparición de daños físicos y psíquicos con importantes consecuencias sobre la salud, problemas laborales y económicos, y disminución del tiempo libre, convirtiendo el cuidado del familiar, percibido anteriormente como motivo de satisfacción, alegría, el sentirse una persona útil, con un alto grado de solidaridad y la ganancia de un enriquecimiento espiritual en su persona, en alguien lleno preocupaciones, cansancio, soledad, depresión, tristeza, ansiedades, asco, constantes sentimientos de irritabilidad, culpa, vergüenza, impotencia y por último frustración. ${ }^{(6)}$

El cuidador es considerado un enfermo silente al acumular una serie de malestares que son enmascarados detrás del alto nivel de exigencia del familiar enfermo, obviando en su empeño la necesidad de su autocuidado para la conservación de su salud.

La creación de espacios académicos, asociado a actividades lúdicas socializadoras dentro de la comunidad, capaces de entregar a estas personas que cuidan de su familiar enfermo con Alzheimer, todo el conocimiento, la compresión, el cariño y el reconocimiento a su labor se pudiera ver como una alternativa para reducir un grupo de dolencias tanto físicas como psicológicas que aparecen en estas personas como consecuencia de la labor que desempeñan. El proyecto recuperador de salud "Respiro de Vida" le dará la posibilidad de disipar ansiedades, miedos y preocupaciones con una ganancia significativa para su salud, 
favorecedora para continuar la práctica de sus cuidados con un conocimiento científicamente fundamentado, un mejor estado físico y la lucidez necesaria ante sus acciones.

Existen diferentes estrategias para recobrar la salud de los cuidadores de personas con Alzheimer ${ }^{(7)}$, sin embargo ninguna ha demostrado total eficacia de manera aislada ${ }^{(5,6)}$, por tanto una idea integradora de estas se muestra tentadora para intentar de alguna manera recuperar la salud del cuidador principal sin alejarse de su comunidad y de su familiar enfermo.

Dada la experiencia de este autor en la orientación y trabajo con este tipo de personas, nos preguntamos si al realizar una estrategia integradora, con los recursos de la comunidad, se pueden observar mejoras en la salud de los cuidadores principales.

De observarse mejoras en la salud de los cuidadores principales permitiría generalizar la experiencia a otros espacios y comunidades. Proponiéndonos determinar su utilidad, como forma de garantizar la recuperación de la salud del cuidador y con ella asegurar su supervivencia y calidad de vida en el familiar enfermo.

\section{Específico.}

1. Caracterizar los sujetos de la investigación.

2. Determinar factores físicos y psicológicos que dañan la salud del cuidador, relacionados con la labor que desempeñan.

3. Valorar la efectividad de un programa integral para cuidadores en la recuperación de su salud física y mental.

4. Evaluar la satisfacción y sus criterios sobre el programa, a través de las de sus participantes.

\section{MÉTODO}

Se desarrolló un estudio descriptivo de corte transversal en la localidad "Los sitios", Centro Habana, de Noviembre de 2009 a Febrero de 2010, aprobándose la inserción de un programa integral para la recuperación de la salud del cuidador principal del enfermo con Alzheimer, dentro del proyecto sociocultural "Quisicuaba", aprovechando las bondades y resultados logrados dentro de la comunidad.

El universo lo conformaron 57 personas que fungían como cuidadores principales de ancianas/os dependientes, dentro del consejo popular 19 "Los Sitios." Relación facilitada por la dirección Municipal de salud a través del responsable del equipo gerontológico de la localidad. Eligiéndose una muestra por conveniencia de 26 cuidadores cruciales, que cumplían los criterios de: cuidar de una persona diagnosticada por estudios clínicos y psicométricos con demencia tipo alzheimer, aprobar su incorporación al programa de recuperación de salud, disponer de una persona sustituta para velar por el familiar enfermo en su ausencia, no recibir remuneración por la actividad que realiza, poder leer y escribir y haberse desempeñado como cuidador de forma permanente por más de un año. Aspectos discutidos y valorados en visita al hogar con consentimiento informado y compromiso oficial del suplente luego de dinámica familiar, junto al resto de los integrantes de la familia.

El programa de recuperación de la salud para cuidadores se desarrolló con una frecuencia semanal de 6 horas, en 16 encuentros, conformado por un grupo de talleres que incluyeron: Actividades físicas con ejercicios bioenergéticos, específicamente Yoga, con selección de 
series para el autocontrol emocional, la disminución de la ansiedad, la depresión u otras alteraciones inhibidoras de la concentración, el descanso y el sueño, capacitación en temas geriátricos y enfermedad de Alzheimer y actividades lúdicas asumidas por aficionados de los talleres culturales del proyecto sociocultural "Quisicuaba" (Manualidades, artes plásticas, música, danza, literatura, cine y arte culinaria)

Para la recogida de información sobre el estado de salud física y su recuperación se utilizaron dinámicas grupales con tormenta de ideas, unificándose por consenso las principales molestias físicas. (Semanas 1 y 14).

Para valorar la salud psicológica y su recuperación se emplearon un grupo de instrumentos validados en nuestro país por el grupo de atención al paciente con demencia y su familiar 10/66 (Cuestionario de sobrecarga de Zarit y zarit, Inventario de autovaloración de ansiedad IDARE, y Escala de depresión de Hamilton) estos indicadores se aplicaron al inicio y en el transcurso del programa. (Semanas 1 diagnóstico y semanas 5, 12 evaluación) Para evaluar nivel de satisfacción y utilidad del programa a criterio de los participantes, se empleó la técnica del PNI, buscando lo positivo, negativo e interesante según criterios de los cuidadores, valorándose además posibles cambios o sugerencias para próximas ediciones.

Para el análisis estadístico se creó una base de datos en Excel, soporte Windows 98 utilizándose la estadística descriptiva para caracterizar la serie de datos, confeccionándose tablas de contingencia para analizar posibles asociaciones entre variables, a través del método no parametrito del Chi cuadrado.

\section{RESULTADOS}

Los sujetos inscritos en el programa de recuperación de la salud, estuvieron representados por 21 mujeres y 5 hombres, con edades promedios para ambos grupos, de 57 a 70 años de edad y una escolaridad del nivel primario $38 \%$, técnico $23 \%$ y universitaria $19 \%$.

Sus funciones como cuidador principal, estuvieron dirigidas principalmente hacia sus padres, $53 \%$ encarnado en la figura materna, luego al vínculo marital (esposos) $34 \%$ y por último los hermanos/as 11\%. Su permanencia en el cuidado, fluctuó ente los 2 y 4 años, $61 \%$, con 5 $23 \%$ y con 6 y más años 15\%. En todos los casos, la persona enferma nunca fue trasladada fuera de su casa o cuidada por otra persona.

El $57 \%$ de los cuidadores no había recibido información anterior sobre la enfermedad de Alzheimer, frente al $42 \%$ quienes hallaron información en revistas y libros prestados por amigos, ninguno de ediciones cubanas.

Los principales problemas físicos, declarados previo a la realización de los ejercicios bioenergéticos, se relacionaron con dolencias en los sistemas osteomuscular 76\% (artralgias, mialgias, pérdida del equilibrio, lumbociatalgías e incapacidad funcional) cardiovascular (dolor precordial, adormecimiento de manos y pies, calambres, dolor en las piernas, palpitaciones), cefalea, inapetencia e insomnio 15\% y gastrointestinal (acidez, vómitos, inapetencia, dolor en epigastrio y constipación) 7.6\%.

Diez semanas más tarde las condiciones físicas de los cuidadores variaron de manera positiva, quedando pendientes por resolver: la inapetencia $15 \%$, el insomnio $11 \%$ y la cefalea $7.6 \%$. 
Entre los factores que ocasionaban alteraciones en la salud psíquica de estas personas, combinamos que el $100 \%$ padecían de ansiedad, asociada a sobrecarga física y psicológica, asociada a algún tipo de depresión $80 \%$.

Valores iniciales en todas las formas de depresión $80 \%$, en los cuidadores, lograron progresos de un $61 \%$ en la quinta semana, y $46 \%$ en la semana 12 , consiguiéndose llegar a la normalidad el 53\% de los sujetos de la investigación. Tabla No 1, Gráfico No 1.

Tabla No 1.

Evaluación de los Estados Depresivos por semanas en Cuidadores principales de la persona con Alzheimer. Proyecto sociocultural "Quisicuaba," Centro Habana. Septiembre - Diciembre del 2009.

\begin{tabular}{|c|c|c|c|c|c|}
\hline Semanas Estado & Normal & $\begin{array}{l}\text { Depresión } \\
\text { Menor }\end{array}$ & $\begin{array}{l}\text { Menos que } \\
\text { depresión } \\
\text { Mayor }\end{array}$ & $\begin{array}{l}\text { Depresión } \\
\text { Mayor }\end{array}$ & $\begin{array}{l}\text { Mas que } \\
\text { depresión Mayor }\end{array}$ \\
\hline Semana 1 & 5 & 10 & 7 & 4 & 0 \\
\hline Semana 2 & 10 & 11 & 3 & 2 & 0 \\
\hline Semana 3 & 14 & 7 & 3 & 2 & 0 \\
\hline
\end{tabular}

Fuente: Escala de depresión de Hamilton.

$\mathrm{p}=0.1975$ (estadísticamente no significativo)

\section{Gráfico No 1.}

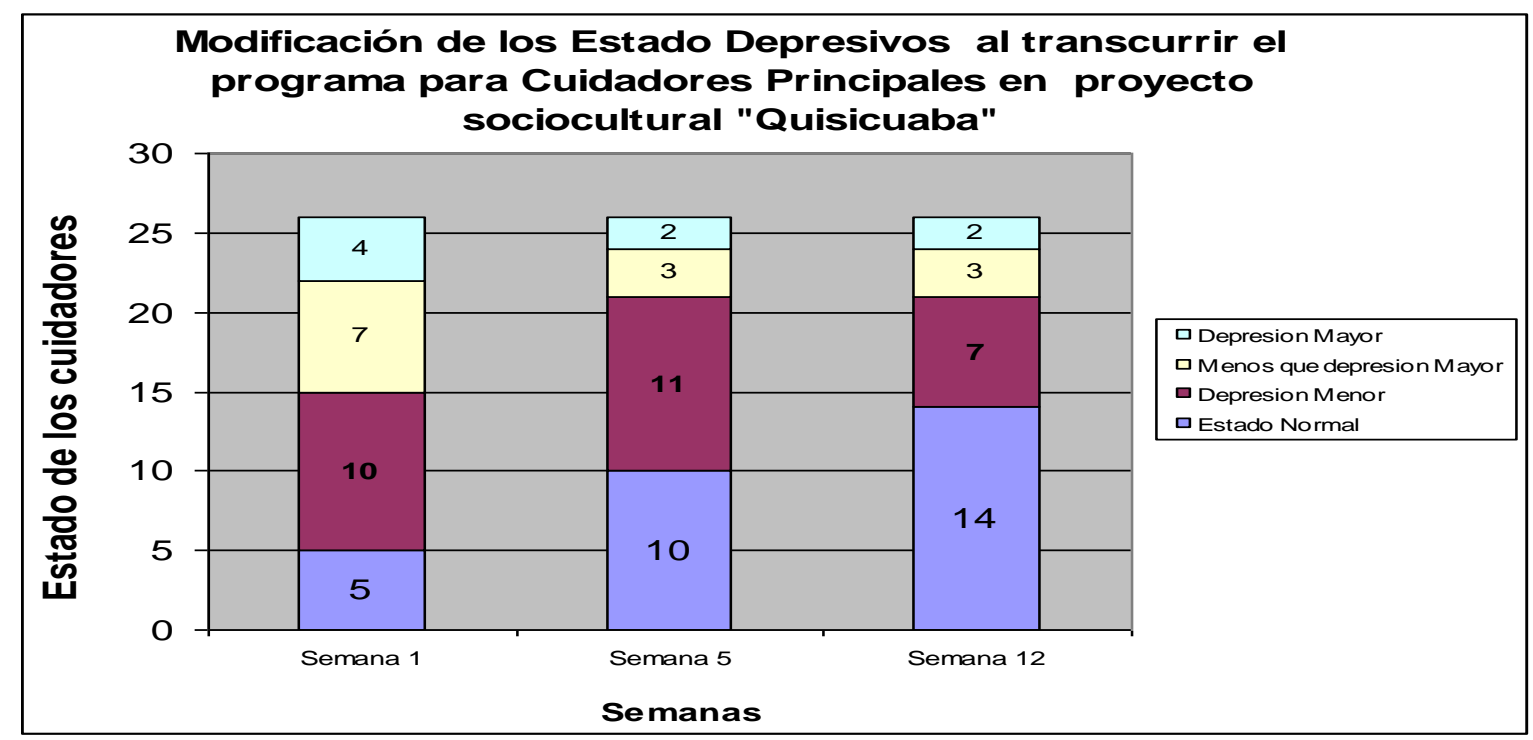

Fuente: Escala de depresión de Hamilton

La ansiedad, considerada como un intenso nerviosismo o inquietud que afecta la capacidad de las personas para funcionar, acompañó al $84 \%$ de los individuos en la primera sesión de trabajo, concibiéndose la forma de estado la de mayor prevalencia en relación a la ansiedad 
de rasgo, la que se mantuvo en dos cuidadores del sexo femenino con dos años en su desempeño.

Gracias al trabajo en equipo y la firmeza de sus participantes por mejorar su salud, los estados de ansiedad alcanzaron mejorados en el transcurso de las semanas, lográndose la rehabilitación del $57 \%$ en la quinta semana y $42 \%$ en la duodécima semana. Tabla No2 y Gráfico No2.

Tabla No2.

Valoración del Estado de ansiedad por semanas en Cuidadores Principales de Personas con Alzheimer. Proyecto sociocultural "Quisicuaba," Centro Habana. Septiembre - Diciembre del 2009.

\begin{tabular}{|l|l|l|l|}
\hline Nivel de Ansiedad & Semana 1 & Semana 2 & Semana 3 \\
\hline Bajo & 4 & 11 & 15 \\
\hline Medio & 18 & 13 & 9 \\
\hline Alto & 4 & 2 & 2 \\
\hline
\end{tabular}

Fuente: Inventario de Ansiedad IDARE.

$\mathrm{p}=0.0364$ (estadísticamente significativo)

\section{Gráfico No 2.}

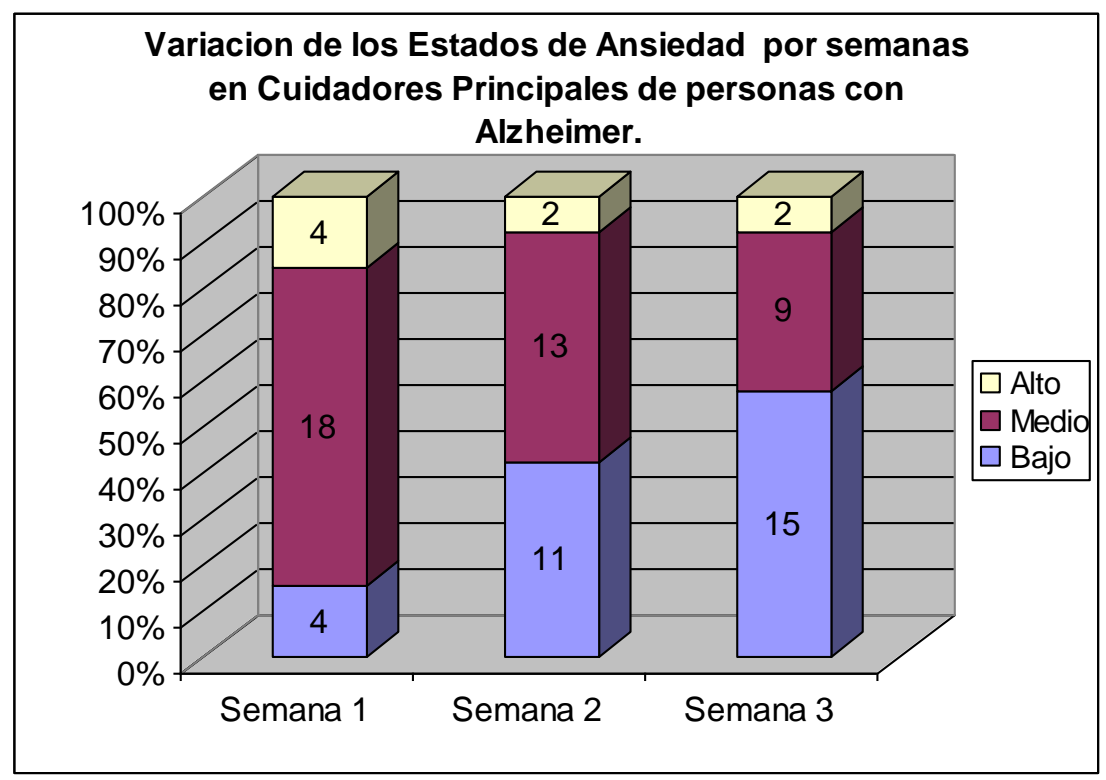

Fuente: Inventario de Ansiedad IDARE

La Sobrecarga física y psicológica, según instrumento, se pudo corroborar en el 100\% de los cuidadores, con supremacía de la sobrecarga intensa 53\% seguida de la moderada $46 \%$ específicamente en la primera semana. Pensándose congruente la permanencia de sobrecarga en evaluaciones posteriores, las que no pudieron ser resueltas, pero si modificadas, al disminuir su intensidad a estados moderados. Tabla No3, Gráfico No3. 
Tabla No 3.

Evaluación de la Sobrecarga física y psicológica del cuidador principal durante su permanencia dentro del progrma. Proyecto sociocultural "Quisicuaba," Centro Habana. Septiembre - Diciembre del 2009.

\begin{tabular}{|c|c|c|c|}
\cline { 3 - 4 } Niveles de & Semana & Semana & Semana \\
Sobrecarga & 1 & 2 & 3 \\
\hline No & & & \\
Sobrecarga & 0 & 0 & 0 \\
\cline { 1 - 1 } Moderada & 12 & 16 & 17 \\
\cline { 1 - 1 } Intensa & 14 & 10 & 9 \\
\hline
\end{tabular}

Fuente: Cuestionario de Sobrecarga Fisica y Psicologica de Zarit-Zarit $p=0.3319$ (estadísticamente no significativo)

\section{Gráfico No 3}

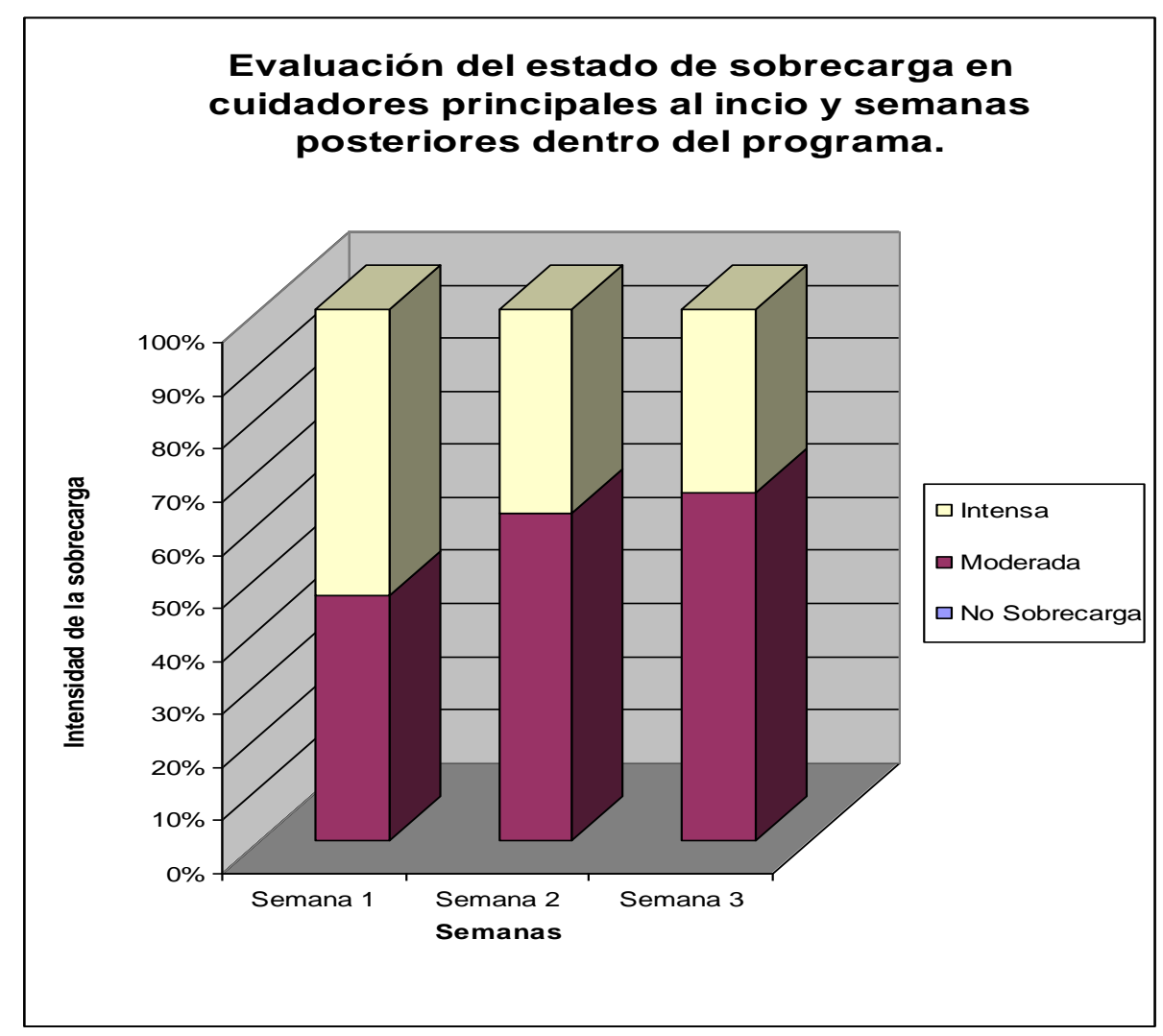

Fuente: Cuestionario de Sobrecarga Fisica y Psicologica de Zarit-Zarit.

Dato interesante, lo constituyó la fuerza de la sobrecarga en relación al tiempo de desempeño como cuidador, estimándose los mayores niveles en personas con permanencia menor o igual a 4 años. Gráfico No 4. 


\section{Gráfico No 4}

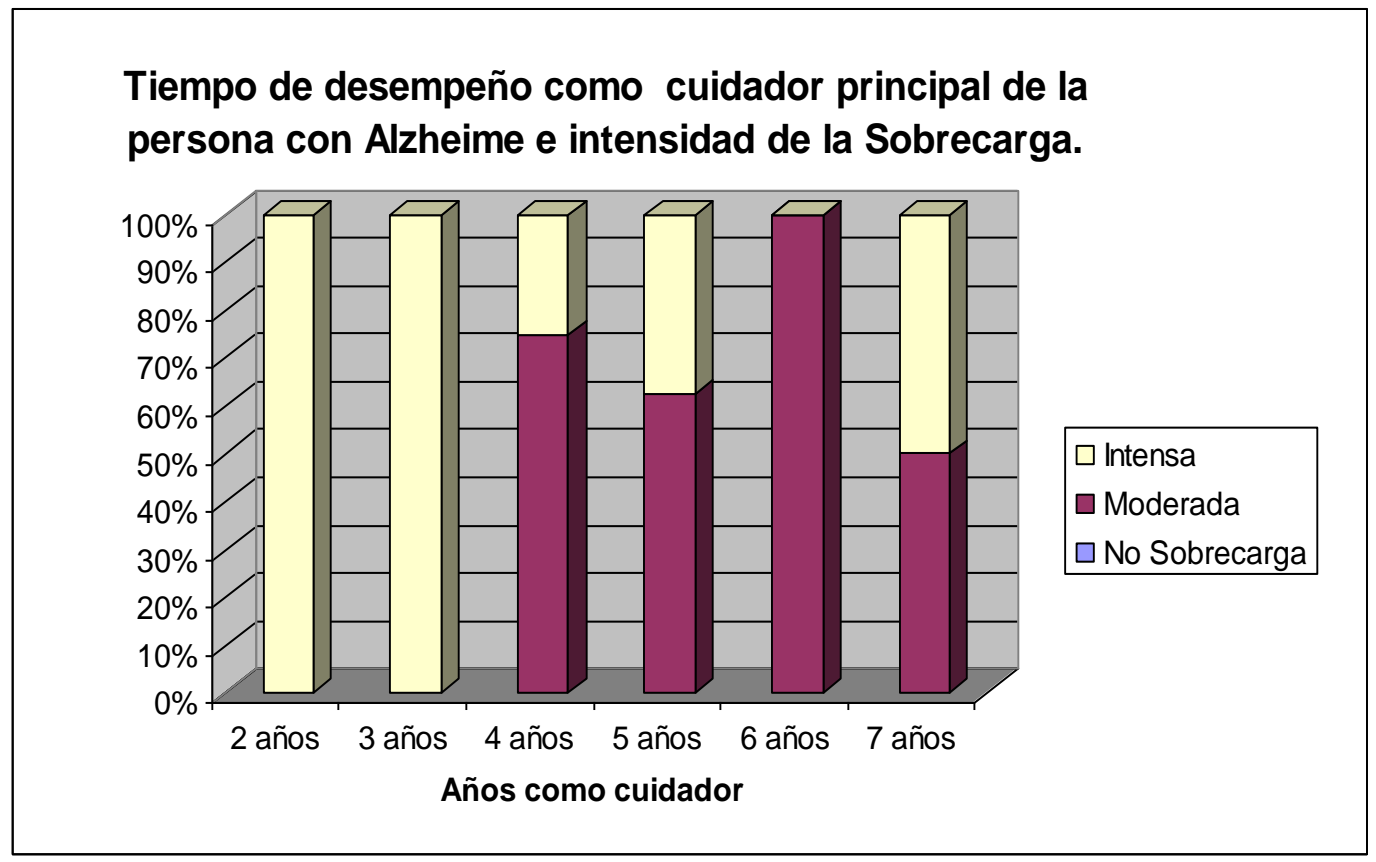

Fuente: Cuestionario de Sobrecarga Fisica y Psicologica de Zarit-Zarit

La valoración final sobre el programa, satisfizo al 100\% de sus participantes, calificándolo de bien $38 \%$ y muy bien $61 \%$. Los aspectos positivos se refirieron a la mejora de su estado físico y psicológico, el poder comprender a su familiar enfermo culpando a la enfermedad por su comportamiento y no a este, al buen trabajo realizado en equipo de profesionales y el disfrute de todas actividades culturales organizadas dentro del proyecto sociocultural "Quisicuaba"

Entre los aspectos negativos señalados se relacionan: las dificultades con los medios de enseñanza audiovisuales, la falta de espacio para la realización de los ejercicios físicos y la complejidad para comprender la enfermedad de Alzheimer.

Como interesante se enunciaron su participación por vez primera en un proyecto sociocultural que le ayudara a rescatar su salud, la ayuda prestada por los niños de la comunidad y los contenidos aprendidos.

Aconsejando que las cesiones de trabajo del programa no deban cerrar, manteniendo la tarea dentro del proyecto, para que otros vecinos de la comunidad y personas en su condición, encuentren el lugar donde nuevamente podrán sentirse persona, considerarse escuchado y aliviar sus penas. Alcanzando concienciar la utilidad de su rol y la importancia de cuidar de su salud.

\section{DISCUSIÓN}

El estudio permitió aproximarnos a las características sociodemográficas de un grupo de cuidadores principales de una persona con Alzheimer, así como el impacto que el desempeño de dicho rol ocasiona sobre su salud física, psíquica y social. La investigación presenta una importante contribución como alternativa inmediata para la recuperación de la 
salud de estas personas relegadas dentro de sus hogares, con un mínimo de recursos y la participación de la comunidad.

Dado que el estudio ha sido realizado en una población muy pequeña, la generalización de sus resultados y conclusiones deben ser analizadas con precaución, aunque creemos que pueden ser extendidos, luego de reconocer que las particulares de los cuidadores son similares a las descritas en otros grupos estudiados. $(\mathbf{7 , 8 , 9 , 1 0 , 1 1 , 1 2 )}$ Así el perfil del cuidador corresponde a una mujer, en edad comprendida entre 57 y 70 años, próxima en ocasiones a la edad de la persona enferma, ama de casa, cónyuge o hija sin otro trabajo fuera del hogar, con pobre solvencia económica para enfrentar exigencias cada vez mayores del familiar y las suyas propias, con una preparación educacional, con predominio del nivel primario, varios técnicos medios y algún universitario. Pocos, tuvieron la posibilidad de recibir información o capacitación en temas relacionados con la enfermedad de Alzheimer, el manejo del familiar enfermo o cómo cuidar de su salud, considerando insuficiente la divulgación de los medios y la preparación de estas personas, antes de instruirse en el programa de recuperador de salud. El no saber manejar a una persona enferma obliga al cuidador a realizar esfuerzos y emplear fuerzas y posiciones que le van provocando ciertas dolencias, reportadas por estos sujetos, las que los cataloga altamente vulnerables para padecer problemas físicos y psíquicos, acercándolos cada vez más al término de "cuidador quemado" con posibilidad de desvanecimiento.

Está claro que el nivel intelectual de un individuo influye en su desenvolvimiento durante el transcurso de su vida, la creatividad, el desarrollo de tareas en el diario vivir, el disfrute de actividades vocacionales y lúdicas, su interés por las relaciones sociales y el mantenimiento de sus capacidades a través de refuerzos educativos, dirigido o autodidacto, posibilitan el mantenimiento de capacidades y/o habilidades para enfrentar ciertas situaciones, las que aún difíciles, pueden ser resueltas $(13,14,15$,

Los síntomas físicos, en su totalidad, están en correspondencia con lo hallado por otros autores, ${ }^{(16,17)}$ apoyando la teoría de la práctica de ejercicios físicos para la recuperación de la salud, particularmente en este grupo, los que en su casi totalidad son ancianos. Se conoce que el concepto de salud en el anciano está dado por varios factores, pero fundamentalmente por su autonomía e independencia ante todas las actividades del diario vivir con la perspectiva más amplia de una integración social adecuada, que es el reflejo de la autonomía social. ${ }^{(15)}$

El validismo, otra manera de llamar a esta independencia, tiene una relación directa con el reflejo del estado mental del individuo, se conoce que llegadas a determinada edad, las personas pueden tener una o más enfermedades crónicas no transmisibles, que al ser atendidas y controladas, les permitirán ser válidos y funcionales, lo que equivale a decir contar con una salud física y mental adecuada. ${ }^{(16,17)}$ Para lograrlo, es importante poner en movimiento nuestro cuerpo. El organismo humano ha sido diseñado para moverse y requiere por tanto realizar ejercicio de forma regular para mantenerse funcional y evitar enfermar. Los movimientos provocados por la actividad física en cualquiera de sus formas, activan músculos y huesos, con beneficios para la salud. Un cuerpo sano se hará acompañar de una mente sana. Las técnicas como el Yoga y Tai Chi, que también incluyen meditación y técnicas de respiración, ofrecen beneficios adicionales para personas ancianas y con enfermedades crónicas, su implementación dentro del programa permitió la disminución y eliminación de importantes dolencias en los cuidadores, ya que al realizar las técnicas bioenergéticas, la corriente de energía del cuerpo se dirige a las zonas que necesitan equilibrarse y así curar la enfermedad y fomentar la salud ${ }^{(16,18)}$ 
La convivencia con una persona aquejada de demencia, trastornos mentales y cambios en su personalidad, promueven en el cuidador principal, estados emocionales enfermizos, efecto de su experiencia ante situaciones estresantes y desconocidas, las que van afectando su salud física y psicológica, acarreando todo tipo de problemas emocionales, los que indudablemente varían de una persona a otra, dependiendo, entre otras cosas, de la percepción sobre la enfermedad, los recursos aprendidos y las habilidades personales para enfrentarse a los problemas. ${ }^{(18,19,20,21)}$

El estado mental del individuo que abarca la esfera cognitiva y afectiva tiene un impacto importante sobre el desenvolvimiento de las habilidades y/o capacidades para su desempeño en el diario vivir. De no protegerse y sobrepasarse los límites admisibles, la persona va iniciando un deterioro progresivo de sus capacidades y/o habilidades, al extremo de sentirse imposibilitado de cumplir sus propias necesidades. La ansiedad, la depresión la sobrecarga y la insuficiente preparación física y psicológica de estas personas exigen una atención inmediata, especial y diferenciada, capaz de protegerlos de daños mayores e incluso de la muerte; análisis coincidente con otros estudiosos del tema, los que también ansían encontrar estrategias inteligentes e inmediatas para este segmento poblacional. (22, $23,24,25,26)$

Una manera práctica, económica y racional de ayudar a personas que cuidan de un enfermo con Alzheimer podría ser la integración y desarrollo de programas educativos, culturales o deportivos dentro de la propia comunidad. La cercanía al enfermo, aunque fuera de casa, la sensación de abandono, el miedo a que algo suceda, la vergüenza de compartir con otros los avatares de sus días, o la desconfianza a no ser comprendido, estarán mejor acordados si socializamos con personas cercanas y en igual condición. Los integrantes de cualquier comunidad cubana, aunque de forma exigua, comparten la vida de sus vecinos, concibiéndolos más sensibles y responsables ante el problema ajeno, la socialización de los males entre personas de buena voluntad, consienten la búsqueda de alternativas alentadoras y vivificantes para los menos favorecidos de la comunidad, haciendo cumplir que "la ayuda entre vecinos consolidad la unidad del barrio."

\section{CONCLUSIONES}

El programa integral recuperador de salud permitió concebir al proyecto sociocultural "Quisicuaba" como espacio de respiro para cuidadores principales, demostrando su utilidad con un mínimo de recursos y el apoyo de profesionales y aficionados del territorio, los que, a través de sus acciones concedieron mejorías a los estados físico y psicológico de sus integrantes, disminuyendo significativamente la ansiedad, mejorando los niveles de depresión y sobrecarga, además de rescatar la autonomía y la autovaloración personal, considerándose oportuna y necesaria su permanencia dentro de la comunidad, como estrategia para otros contiguos en igual condición, él que hallará paz, comprensión y salud muy cerca de su casa.

\section{BIBLIOGRAFÍA}

1. Spielberger CD,Gorsuch RL, Lushene RE.Cuestionario de Ansiedad EstadoRasgo.Manual, 2ª ed. Madrid: TEA Ediciones, 1986

2. Grau J.A. y Martín M (1993): Estrés, ansiedad, personalidad. Resultados de las investigaciones cubanas realizadas con el enfoque personal. Rev. Interamericana de Psicología 27 (1), 37-58

3. Castellanos B, Grau J.A. y Martín M (1986): Caracterización de la ansiedad personal presente en la personalidad premórbida de pacientes con TTS y neurosis de 
ansiedad. Trabajo de Diploma, Facultad de Psicología, Universidad Central de las Villas.

4. Alzheimer's Disease International (1999). The prevalence of dementia [Factsheet 3]. Abril de 2004. [Citado el 21 de enero de 2008]. Disponible en: www.alz.co.uk/adi/pdf/3preval.pdf

5. Roca Socarras A., Blanco Torres K. Carga en familiares cuidadores de ancianos dementes. [Consultado el 11 Marzo del 2010] Disponible en:

http://www.cocmed.sld.cu/no114sp/ns114rev4.htm.

6. Reflexión en torno a cuidadores a la atención a los cuidadores informales de personas con demencia y propuesta de una intervención interdisciplinaria. Psicología conductual, vol.15, No 1, 2007, pp.57-76. [Consultado el 11 Marzo del 2010 ] Disponible en: http://areas.cs.urjc.es/psicologia/www/cuidar cuidandose.pdf. 7. Rodríguez Rodríguez P. "El apoyo informal en la provisión de cuidados a las personas con dependencias. Una visión desde el análisis de género", en Ley de Dependencia y Educación Infantil como medidas de conciliación de la vida laboral y familiar. Forum de Política Feminista. Madrid. 2004.

8. Bover Bover, A. Cuidadores informales de salud del ámbito domiciliario: percepciones ligadas al género y a la generación. [tesis]. Illes Balears: Universitat de les Illes Balears; 2004. [Citado el 10 noviembre del 2005] Disponible en:

http://www.tdx.cesca.es.

9. Formación de familias cuidadoras de personas mayores dependientes.

[Consultado el 16 Enero del 2010] Disponible en:

http://www.ucm.es/BUCM/revistas/trs/02140314/articulos/CUTS9898110203A.PDF

10. El rol del cuidador de las personas dependientes y sus repercusiones sobre su calidad de Vida y su salud. [Consultado el 18 Febrero del 2010] Disponible en:

http://scielo.isciii.es/scielo.php?pid=S1699-

695X2009000200004\&script=sci arttext\&tlng=en

11. Impacto psicosocial del síndrome demencial en cuidadores. [Consultado el 11 Febrero del 2010] Disponible en:

http://www.bvs.sld.cu/revistas/mgi/vol24 1 08/mgi05108.htm

12. Características sociodemográficas, de salud y utilización de recursos sanitarios de cuidadores de ancianos atendidos en domicilio. [Consultado el 13 de Enero del 2010] Disponible en: http://scielo.isciii.es/scielo.php?pid=S1134-

928X2009000100003\&script $=$ sci arttext\&tlng $=\mathrm{e}$

13. Gálvez J. Ras E, Hospital I, Vila A. Perfil del cuidador principal y valoración del nivel de ansiedad y depresión. Aten Primaria 2003; 31: 144-145.

14. Peinado, A.I., Garcés, E.J. (1998) Burnout en cuidadores principales de pacientes con Alzheimer: Síndrome del asistente desatendido. Anales de Psicología, 14(1), 8393.

15. Moreno Gaviño I. Bernabeu Wittel M, Álvarez Tello M, Rincón Gómez, M.,Bohórquez Colombo P.,Cassani Garza M et al. Sobrecarga sentida por la figura del cuidador principal en una cohorte de pacientes pluripatológicos. Aten primaria 2008; 40: 193-198.

16. Ejercicios, su importancia para toda la vida. Congreso Red de sociedades Científicas VI encuentro 13,14 y14 julio 2004.

17. Solé Carrasco L. Tesis para optar por el grado de master en longevidad satisfactoria. Ejercicio físico y salud en un grupo de adultos mayores pertenecientes al área del Policlínico Docente Universitario”Dr. Carlos J. Finaly" Municipio Marianao, 2007.

18. Programas e intervenciones de apoyo a los cuidadores informales en España. [Consultado el 10 de Enero del 2010] Disponible en 
http://scielo.isciii.es/scielo.php?pid=S1134-

928X2008000100002\&script=sci arttext\&tlng=en

19. González, J. Armengol, J., Cuixart, I., Manteca, H. Carbonell, M.A. (2005) Cuidar a los cuidadores: Atención Familiar. VI congreso Virtual de Psiquiatría, Ínter psiquis 2005.

20. Informales de adultos mayores con demencia. Rev. Cub. Salud

publica vol.35 no.2 La Habana Apr./Jun 2009.

21. Síndrome del cuidador de adultos mayores discapacitados y sus implicaciones

Psicosociales. [Consultado 23 Diciembre del 2009 ] Disponible en:

http://www.scielo.org.co/scielo.php?pid=S1657-

$95342006000500006 \&$ script=sci arttext\&tlng=es.

22. Experiencias en la intervención psicológica con familias de personas dependientes

[Consultado el 10 de Enero del 2010] Disponible en:

http://scielo.isciii.es/scielo.php?pid=S1132-

$05592007000100008 \&$ script $=$ sci arttext\&tlng $=$ es.

23. Orientación para una mejor atención de los ancianos con Alzheimer en la comunidad. [Consultado el 2 de Marzo del 2010] Disponible en: http://scielo.sld.cu/scielo.php?pid=S1729-

519X2008000400021\&script=sci arttext\&ting=pt.

24. ¿Quien cuida al cuidador? [Consultado el 10 de Enero del 2010] Disponible en http Espin Andrade AM. "Escuela de cuidadores" como programa psicoeducativo para cuidadores://www.revistahospitalarias.org/info 2003/02 172 14.htm.

25. Ansiedad y depresión en familiares y cuidadores de pacientes con enfermedad de Alzheimer. [Consultado el 19 de Marzo del 2010] Disponible en:

http://www.medicosypacientes.com/noticias/2009/06/09 0627 alzheimer.

26. Cuidar a un enfermo de Alzheimer puede provocar depresión y ansiedad. [Consultado el 19 de Marzo del 2010] Disponible en:

http://www.informador.com.mx/jalisco/2009/135238/6/cuidar-a-un-enfermo-de-

alzheimer-puede-provocar-depresion-y-ansiedad.htm.

27. El apoyo social como modulador del cuidador como modulador de la carga del cuidador de enfermos con Alzheimer.[Consultado el 18 Febrero del 2010] Disponible en:

http://redalyc.uaemex.mx/redalyc/pdf/291/29115104.pdf.

28. Intervención con cuidadores de familiares mayores dependientes: una revisión. [Consultado el 19 de Marzo del 2010] Disponible en:

http://www.psicothema.com/pdf/3330.pdf

29. Cuidar a una persona con alzheimer provoca ansiedad, insomnio, depresión o un aumento del consumo de alcohol y de sustancias tóxicas. [Consultado el 19 de Marzo del 2010] Disponible en:

http://www.noticiasmedicas.es/medicina/noticias/172/1/Cuidar-a-una-persona-conalzheimer-provoca-ansiedad-insomnio-depresion-o-un-aumento-del-consumo-dealcohol-y-de-sustancias-toxicas/Page1.html.

30. Depresión en cuidadores informales. [Consultado el 13 de Marzo del 2010] Disponible en: http://www.puce.edu.ec/documentos/Medicinav5.pdf\#page=63.

31. Guerra Hernández M. Síntomas Psicológicos y conductuales en pacientes con demencia. Rev. Haban cienc méd La Habana, Vol. VIII No. 1,ene-mar 2009. 